\title{
Infektiöse Leiche
}

\section{Obduktion endete auf der Krankenstation}

\begin{abstract}
Dass Tote einem nichts tun, trifft in den meisten Fällen zu. All zu sicher sollten sich Mitarbeiter der Rechtsmedizin oder Pathologie aber nicht fühlen, denn völlig ungefährlich ist der Umgang mit Verstorbenen nicht. So mag zwar der Patient tot, die Keime in und auf seinem Körper aber noch am Leben sein, wie der Fall von Dr. Lars Hagemeier und seinen Kollegen zeigt.
\end{abstract}

Vier Tage nach ihrem Tod wurde die Leiche einer 31-Jährigen in die Pathologie gebracht. Drei Wochen zuvor war die Frau mit einer offenen Fußverletzung aus Australien heimgekehrt. Die Verletzung hatte sie sich auf einer Farm zugezogen, und da sich die Wunde entzündet und sie hohes Fieber hatte, brach sie ihren Urlaub frühzeitig ab. Doch auch in Deutschland ließen die Symptome nicht nach. Mit Übelkeit und Kopfschmerzen begab sie sich in ein Krankenhaus, außerdem litt sie unter progredienten Schmerzen und Krämpfen in Beinen und Füßen. Ihr Blutbild war unauffällig. Nach einer überstandenen nächtlichen Herz-Kreislauf-Dekompensation mit Blutdruckabfall klagte sie dann morgens über Atemnot. Das Labor stellte eine Myolyse fest. Knapp zwei Stunden später starb die Frau auf der neurologischen Intensivstation mit Anzeichen einer schwerwiegenden Sepsis.

\section{Fortgeschrittene Fäulnis und Gasentwicklung}

Trotz Kühlung fanden sich an ihrem Leichnam bereits zu Beginn der Obduktion fortgeschrittene Fäulnisveränderungen: Die Haut am oberen Thorax war grün verfärbt, die Venennetze an den Extremitäten waren durchgeschlagen, die Hals- und obere Brustmuskulatur grünlich-sulzig verändert. In der Haut des oberen Brustkorbs hatte sich
Gas gebildet, die Leber war schaumig aufgegangen. Nähere neuropathologische Untersuchungen brachten hyaline Thromben in den Blutgefäßen und Fäulnisblasen in den Stammganglien ans Licht. Mikroskopisch zeigten sich im Gehirngewebe heterogen zusammengesetzte Bakterienhaufen mit einer grampositiven und -negativen Mischflora. Alles in allem schien ein finales Schockgeschehen den Tod der Patientin verschuldet zu haben. Den Grund lieferten die Abstriche: hämolysierende Streptokokken der Gruppe A.

\section{Waren Streptokokken in der Atemluft?}

Vier Tage nach der Obduktion erkrankte ein Mitarbeiter der Pathologie an einer beidseitigen Lungenentzündung. Die Ursache fanden die Ärzte in der Blutkultur: Streptokokken der Gruppe A.

Dass von Leichen eine Infektionsgefahr ausgeht, ist durchaus bekannt. Sowohl Bakterien als auch Viren können bei der Obduktion aerogen übertragen werden. Die Infektiosität einer Leiche hängt dabei von der Art des Erregers ab. Während die mittlere Überlebenszeit von Viren auf unbelebten Oberflächen zwischen Stunden (Influenza-, Hepatitis-A-Viren) und Tagen (Noroviren, Hepatitis-B-Viren, HIV) liegt, können Bakterien wie Staphylococcus aureus und Enterokokken mehrere Wochen
Angst vor einer Leiche? Mit unter zu Recht, wenn fiese Keime im Spiel sind.

persitieren. Werden sie aufgewirbelt zum Beispiel durch eine oszillierende Säge, gelangen sie in die Atemluft. Ebenso können freigesetzte Fäulnisgase Toxine und Bakterien enthalten. Ein Mundnasenschutz sowie Schutzhandschuhe sollten deshalb immer getragen werden. Und auch die sorgfältige Händedesinfektion nach Ablegen der Schutzhandschuhe empfiehlt Hagemeier. Von den an der Zersetzung beteiligten Mikroorganismen geht hingegen in der Regel keine Gefahr aus.

Ob sich der Mitarbeiter im beschriebenen Fall am Leichnam angesteckt hatte, konnte letztendlich nicht sicher geklärt werden. Zwar stimmte sein Antibiogramm mit dem des Leichnams überein, doch die Typisierung des für die Pneumonie verantwortlichen Bakterienstamms fiel der frühzeitigen Vernichtung der Rückstellprobe im Krankenhaus zum Opfer. (Christopher Heidt) Rechtsmedizin 2010, 21:131 